\title{
GLOBAL BIFURCATION THEOREMS FOR NONCOMPACT OPERATORS
}

\author{
BY JOHN MACBAIN
}

Communicated by Michael Golomb, February 24, 1974

1. Introduction. The first general existence theorem for bifurcation points was obtained by Krasnoselski [1]. He considered the equation $u=\lambda L u+H(\lambda, u)$ in a real Banach space $\mathscr{B}$ where $L$ and $H$ are compact, and $H$ is $o(\|u\|)$ uniformly on each bounded $\lambda$ interval for small $u$. In this situation he proved that if $\lambda$ is a characteristic value of $L$ having odd multiplicity, then $(\lambda, 0)$ is a bifurcation point in $R \times \mathscr{B}$. Much more recently, Rabinowitz [2] considered the same problem and, using a LeraySchauder degree argument, obtained a two-fold alternative for the global behavior of these bifurcation branches.

This paper extends the results of Krasnoselski and Rabinowitz to a much larger class of operator equations. First to be considered is the equation

$$
L u=\lambda u+H(\lambda, u)
$$

in a real Hilbert space $\mathscr{H}$, where $H$ is as above and $L$ is selfadjoint (bounded or unbounded). In this case, each isolated eigenvalue of $L$ having odd multiplicity is a bifurcation point possessing a continuous branch. Moreover, an alternative theorem on the global behavior of these branches is obtained.

By use of similar arguments these results for selfadjoint operators are extended to a general class of linear operators in a real Banach space $\mathscr{B}$.

2. The selfadjoint operators. In this section all work is in a real Hilbert space $\mathscr{H}, L$ is a selfadjoint operator taking $\mathscr{H}$ into $\mathscr{H}$, and $H(\lambda, u)$ is a compact operator taking $R \times \mathscr{H}$ into $\mathscr{H}$ that is $o(\|u\|)$ uniformly on each bounded $\lambda$ interval for small $u$.

Let $\mathscr{E}$ denote $R \times \mathscr{H}$ with the product topology. For $\mathscr{V} \subset \mathscr{E}$, a subcontinuum of $\mathscr{V}$ is a subset of $\mathscr{V}$ which is closed and connected in $\mathscr{E}$. The trivial solutions of $(1)$ are the points $(\lambda, 0)$, and all other solutions are called nontrivial. Let $\mathscr{S}$ denote all nontrivial solutions of (1), and let $\mathscr{C}_{\lambda_{0}}$ denote the maximal subcontinuum of $\mathscr{S} \cup\left(\lambda_{0}, 0\right)$ containing $\left(\lambda_{0}, 0\right)$.

AMS (MOS) subject classifications (1970). Primary 47H15, 46N05.

Key words and phrases. Nonlinear operator equations, bifurcation.

Copyright (c) American Mathematical Society 1974 
For a subset $A$ of $R, \mathscr{H}$, or $\mathscr{E}, \mathrm{Cl}(A)$ denotes its closure in the respective space. For $A \subset \mathscr{E}, A_{R}$ denotes $\{\lambda \mid(\lambda, u) \in A$ for some $u\}$, and $A \mathscr{H}$ denotes $\{u \mid(\lambda, u) \in A$ for some $\lambda\}$. By an isolated eigenvalue $\lambda$ of $L$, we mean that $\lambda$ is an eigenvalue of $L$ and $\operatorname{dist}(\lambda, \operatorname{sp} L \backslash \lambda)>0$.

The following lemma is stated without proof.

LEMma 1. Suppose $\lambda_{0}$ is an isolated eigenvalue of $L$ having finite multiplicity. Assume $\mathscr{C}_{\lambda_{0}}$ is bounded, $\mathrm{Cl}\left(\left(\mathscr{C}_{\lambda_{0}}\right)_{R}\right) \cap$ ess sp $L=\varnothing$, and $\mathscr{C}_{\lambda_{0}} \cap$ $\{R \times\{0\}\}=\left(\lambda_{0}, 0\right)$. Then $\mathscr{C}_{\lambda_{0}}$ is compact and there exists a bounded open set $\mathscr{O} \subset \mathscr{E}$ such that $\mathscr{C}_{\lambda_{0}} \subset \mathcal{O}, \partial \mathcal{O} \cap \mathscr{S}=\varnothing, \mathrm{Cl}\left(\left(\mathcal{O}_{R}\right)\right)$ ness $\mathrm{sp} L=\varnothing$, the only trivial solutions contained in $\mathcal{O}$ are points $(\lambda, 0)$ where $\left|\lambda-\lambda_{0}\right|<\varepsilon$ for some $\varepsilon<\varepsilon_{0}=\operatorname{dist}\left(\lambda_{0}, \operatorname{sp} L \backslash \lambda_{0}\right)$, and $\operatorname{dist}(\partial \mathcal{O},\{\operatorname{sp} L \times\{0\}\}) \geqq 2 \varepsilon_{1}$ for some positive $\varepsilon_{1}$.

REMARK. The theorem below will show that the hypotheses of the preceding lemma imply that $\lambda_{0}$ is an eigenvalue of even multiplicity.

THEOREM 1. Let $\lambda_{0}$ be an isolated eigenvalue of $L$ having odd multiplicity. Then

(i) $\mathscr{C}_{\lambda_{0}}$ is unbounded, or

(ii) $\mathscr{C}_{\lambda_{0}}$ is bounded and $\mathrm{Cl}\left(\left(\mathscr{C}_{\lambda_{0}}\right)_{R}\right)$ ness sp $L \neq \varnothing$, or

(iii) $\mathscr{C}_{\lambda_{0}}$ is compact, $\mathrm{Cl}\left(\left(\mathscr{C}_{\lambda_{0}}\right)_{R}\right) \cap$ ess sp $L=\varnothing$, and $\mathscr{C}_{\lambda_{0}}$ contains trivial solutions other than $\left(\lambda_{0}, 0\right)$.

Proof. Let us define $\Phi(\lambda, u)=L u-\lambda u-H(\lambda, u)$. In general, degree theory cannot be applied to such an operator. Under the hypothesis on $L$ we will show how $\Phi$ can be replaced by a compact perturbation of the identity, thus allowing the use of degree theory.

Assume that none of (i), (ii), and (iii) occurs. Then by Lemma 1 we find a bounded open set $\mathcal{O}, \varepsilon>0$, and $\varepsilon_{1}>0$, such that $\mathscr{C}_{\lambda_{0}} \subset \mathcal{O}, \mathrm{Cl}\left(\left(\mathcal{O}_{R}\right)\right) \cap$ ess sp $L=\varnothing, \partial \mathcal{O} \cap \mathscr{S}=\varnothing, \operatorname{dist}(\partial \mathcal{O},\{\operatorname{sp} L \times\{0\}\}) \geqq 2 \varepsilon_{1}$, and the only trivial solutions to (1) in $\mathcal{O}$ are points $(\lambda, 0)$ satisfying $\left|\lambda-\lambda_{0}\right|<\varepsilon<\varepsilon_{0}$, where $\varepsilon_{0}=\operatorname{dist}\left(\lambda_{0}, \operatorname{sp} L \backslash\left\{\lambda_{0}\right\}\right)$.

Select a neighborhood $N$ of ess sp $L$ which contains $\mathrm{Cl}\left(\left(\mathcal{O}_{R}\right)\right)$ in its exterior, and let $\mu_{0} \notin \mathrm{Cl}\left(\left(\mathcal{O}_{R}\right)\right)$ be in the resolvent set. Let $\mathscr{H}^{\prime}$ denote the maximal closed subspace for which $L \mathscr{H}^{\prime} \subseteq \mathscr{H}^{\prime}$ and $\operatorname{sp} L \mid \mathscr{H}^{\prime}=\operatorname{sp} L \cap N$, and let $P$ be the projector onto $\mathscr{H}^{\prime}$. Define the linear operator $L_{0}$ by

$$
L_{0}=\left(L-\mu_{0} I\right)(I-P) .
$$

$L_{0}$ is clearly compact. Furthermore, $\lambda \notin N$ is an eigenvalue of $L$ having multiplicity $m$ if and only if $\lambda-\mu_{0}$ is an eigenvalue of $L_{0}$ having multiplicity $m$. For $\lambda \notin\left\{\mu_{0}\right\} \cup N$ we define

$$
G_{\lambda}=\left(\lambda-\mu_{0}\right)^{-1}\left[L_{0}+(I-P)(-H(\lambda, u))\right]+(\lambda-L)^{-1} P(-H(\lambda, u)) .
$$


From the definition of $P$ it follows that (1) is equivalent to

$$
u=G_{\lambda} u
$$

for $\lambda$ in a neighborhood of $\mathrm{Cl}\left(\left(\mathcal{O}_{R}\right)\right)$. The linear part of $G_{\lambda}$ is compact and the linear part of $G_{\lambda_{0}}$ has the eigenvalue 1 with multiplicity $m_{0}$ if and only if $L$ has the eigenvalue $\lambda_{0}$ with multiplicity $m_{0}$. The nonlinear part of $G_{\lambda}$ is also compact and in norm is $o(\|u\|)$ for small $u$.

(2) is the form necessary for the use of Leray-Schauder degree theory. Applying this theory as Rabinowitz [2] did shows that one of (i), (ii), or (iii) must occur.

REMARK. If the multiplicity of $\lambda_{0}$ is odd, Theorem 1 guarantees that $\lambda_{0}$ is a bifurcation point with a continuous branch $\mathscr{C}_{\lambda_{0}}$.

COROLLARY 1. Let $\lambda_{0}$ be an isolated eigenvalue of $L$ of finite multiplicity which is a bifurcation point with continuous branch $\lambda_{0}$. Then

(i)' $\mathscr{C}_{\lambda_{0}}$ is unbounded, or

(ii)' $\mathscr{C}_{\lambda_{0}}$ is bounded and $\mathrm{Cl}\left(\left(\mathscr{C}_{\lambda_{0}}\right)_{R}\right) \cap$ ess sp $L \neq \varnothing$, or

(iii) $^{\prime} \mathscr{C}_{\lambda_{0}}$ is compact, $\operatorname{Cl}\left(\left(\mathscr{C}_{\lambda_{0}}\right)_{R}\right) \cap \mathrm{sp} L=\left\{\lambda_{0}, \lambda_{1}, \cdots, \lambda_{n}\right\}$ and the sum of the multiplicities of the eigenvalues $\lambda_{0}, \lambda_{1}, \cdots, \lambda_{n}$ is even.

We now consider

$$
L u=\lambda K u+H(\lambda, u)
$$

where $K$ is positive definite and bounded and $L, H$ are as above.

COROllaRY 2. Let $R$ be the positive square root of $K$. Let $\lambda_{0}$ be an isolated eigenvalue of $R^{-1} L R^{-1}$ of finite multiplicity which is a bifurcation point of (3) with a continuous branch $\mathscr{D}_{\lambda_{0}}$. Then

(i) $\mathscr{D}_{\lambda_{0}}$ is unbounded, or

(ii) $\mathscr{D}_{\lambda_{0}}$ is bounded and $\mathrm{Cl}\left(\left(\mathscr{D}_{\lambda_{0}}\right)_{R}\right) \cap \operatorname{ess} \operatorname{sp}\left(R^{-1} L R^{-1}\right) \neq \varnothing$, or

(iii) $\mathscr{D}_{\lambda_{0}}$ is compact, $\operatorname{Cl}\left(\left(\mathscr{D}_{\lambda_{0}}\right)_{R}\right) \cap \operatorname{sp}\left(R^{-1} L R^{-1}\right)=\left\{\lambda_{0}, \lambda_{1}, \cdots, \lambda_{n}\right\}$ and the sum of the multiplicities of the eigenvalues $\lambda_{0}, \lambda_{1}, \cdots, \lambda_{n}\left(\right.$ of $\left.R^{-1} L R^{-1}\right)$ is even.

If the multiplicity of $\lambda_{0}$ is odd, then $\left(\lambda_{0}, 0\right)$ is a bifurcation point possessing a continuous branch.

3. General operators. We now generalize by considering a real Banach space $\mathscr{B}$ and linear operators $T: \mathscr{B} \rightarrow \mathscr{B}$. The equation being studied is

$$
T u=\lambda u+H(\lambda, u)
$$

with $H$ as before. 
THEOREM 2. Suppose $\lambda_{0}$ is an isolated eigenvalue of $T$ of odd multiplicity and

(a) to every closed interval $\sigma \subset R \mid$ ess sp $T$ containing $\lambda_{0}$ there is a compact projector $Q_{\sigma}$ that commutes with $T$, and $\lambda_{0}$ is an isolated eigenvalue of $T \mid Q_{\sigma} \mathscr{B}$ of odd multiplicity,

(b) the restriction of $T-\lambda I$ to $\left(I-Q_{\sigma}\right) \mathscr{B}$ is invertible for $\lambda \in \sigma$.

Then $\left(\lambda_{0}, 0\right)$ is a bifurcation point possessing a continuous branch $\mathscr{C}_{\lambda_{0}}$ such that

(i) $\mathscr{C}_{\lambda_{0}}$ is unbounded, or

(ii) $\mathscr{C}_{\lambda_{0}}$ is bounded and $\mathrm{Cl}\left(\left(\mathscr{C}_{\lambda_{0}}\right)_{R}\right)$ ness sp $T \neq \varnothing$, or

(iii) $\mathscr{C}_{\lambda_{0}}$ is compact, $\left(\mathscr{C}_{\lambda_{0}}\right)_{R} \cap \mathrm{sp} T=\left\{\lambda_{0}, \lambda_{1}, \cdots, \lambda_{n}\right\}$ and the sum of the multiplicities of the eigenvalues $\lambda_{0}, \lambda_{1}, \cdots, \lambda_{n}$ is even.

Proof. The proof is similar to that of Theorem 1.

Corollary 3. Suppose $\lambda_{0}$ is an isolated eigenvalue of $T$ of odd multiplicity and for every closed interval $\sigma \subset R \backslash$ ess sp $T$ containing $\lambda_{0}, T$ can be uniformly approximated by operators $T_{\varepsilon}$ which are of the type treated in Theorem 2 and such that $\operatorname{sp} T_{\varepsilon} \cap \sigma=\operatorname{sp} T \cap \sigma$ up to multiplicity of eigenvalues. Then the results of Theorem 2 hold for $T$ and $\mathscr{C}_{\lambda_{0}}$.

Our work necessitates the use of a complexification of $\mathscr{B}$ which is denoted by $\hat{\mathscr{B}}=\mathscr{B} \times \mathscr{B}$. The general element of $\hat{\mathscr{B}}$ is

$$
(x, y)=x+i y \quad \text { and } \quad\|(x, y)\|_{\hat{B}}=\left(\|x\|^{2}+\|y\|^{2}\right)^{1 / 2} \text {, }
$$

where $\|\cdot\|$ is the norm in $\mathscr{B}$. For any linear $T: \mathscr{B} \rightarrow \mathscr{B}, \hat{T}: \hat{\mathscr{B}} \rightarrow \hat{\mathscr{B}}$ is its unique linear extension to $\hat{\mathscr{B}}$.

THEOREM 3. Let $T$ be a bounded linear operator and $\sigma$ be a compact subset of $R \backslash$ ess sp $\hat{T}$. Then there is a bounded projector $Q_{\sigma}$ that commutes with $T$ such that the restriction of $T-\lambda I$ to $\left(I-Q_{\sigma}\right) \mathscr{B}$ is invertible for $\lambda \in \sigma$ and $Q_{\sigma} \mathscr{B}$ is the span of the principal manifolds belonging to eigenvalues of $T$ in $\sigma$.

Proof. The first step is to go to the complexifications $\hat{T}$ and $\mathscr{B}$. A decomposition theorem [3] is applicable to this complex case. From this complex decomposition, we can derive suitable real projections from $\mathscr{B}$ into $\mathscr{B}$ and their corresponding subspaces in $\mathscr{B}$.

REMARK. It follows from this theorem that Theorem 2 holds for all bounded linear operators $T$ on $\mathscr{B}$ for which $R \cap$ ess sp $\hat{T}=\operatorname{ess} \operatorname{sp} T$. In particular this is true if $T$ is compact, or if $\mathscr{B}$ is a Hilbert space and $T$ is selfadjoint. 


\section{REFERENCES}

1. M. A. Krasnosel'skiǐ, Topological methods in the theory of nonlinear integral equations, GITTL, Moscow, 1956; English transl., Macmillan, New York, 1964. MR 20 \#3464; 28 \#2414.

2. P. H. Rabinowitz, Some aspects of nonlinear eigenvalue problems, Rocky Mountain J. Math. 3 (1973), no. 2, 161-202.

3. F. Riesz and B. Sz.-Nagy, Leçons d'analyse fonctionnelle, 2nd ed., Akad. Kiadó, Budapest, 1953; English transl., Functional analysis, Ungar, New York, 1971. MR 15, 132.

Department of Mathematics, Purdue University, West Lafayette, Indiana 47907

Current address: Air Force Institute of Technology, Wright-Patterson Air Force Base, Dayton, Ohio 45433 